\title{
LETTER \\ Combining Attention Model with Hierarchical Graph Representation for Region-Based Image Retrieval
}

\author{
Song-He FENG ${ }^{\dagger \mathrm{a})}$, Student Member, De $\mathrm{XU}^{\dagger \mathrm{b})}$, Member, and Bing $\mathrm{LI}^{\dagger \mathrm{c})}$, Student Member
}

\begin{abstract}
SUMMARY The manifold-ranking algorithm has been successfully adopted in content-based image retrieval (CBIR) in recent years. However, while the global low-level features are widely utilized in current systems, region-based features have received little attention. In this paper, a novel attention-driven transductive framework based on a hierarchical graph representation is proposed for region-based image retrieval (RBIR). This approach can be characterized by two key properties: (1) Since the issue about region significance is the key problem in region-based retrieval, a visual attention model is chosen here to measure the regions' significance. (2) A hierarchical graph representation which combines region-level with image-level similarities is utilized for the manifold-ranking method. A novel propagation energy function is defined which takes both low-level visual features and regional significance into consideration. Experimental results demonstrate that the proposed approach shows the satisfactory retrieval performance compared to the global-based and the block-based manifold-ranking methods.

key words: region-based image retrieval, manifold-ranking, hierarchical graph representation, visual attention model
\end{abstract}

\section{Introduction}

Content-based image retrieval (CBIR) has been widely investigated in the past few years, and much effort has been devoted to bridging the semantic gap [3]. More recently, the manifold-ranking algorithm [6], which can effectively explore the relationship among all the samples, has been successfully integrated into CBIR [4]. The manifold-ranking algorithm is able to rank data points with only positive training samples. It can be intuitively understood as a process of spreading labels from positive training samples to unlabeled samples. In essence, manifold-ranking algorithm is a graphbased semi-supervised method and of course it belongs to the transductive methods since it aims to accurately predict the relevance of unlabeled images which are attainable during the training stage.

[4] takes each image as a vertex in a weighted graph and propagates the ranking score of labeled examples by using the manifold-ranking algorithm, which has shown promising retrieval results on the Corel dataset. However, [4] suffers from the limitation that the algorithm uses the global-based features to denote the image content, which is deemed to be inappropriate to reflect the intrinsic global

Manuscript received January 15, 2008.

Manuscript revised March 30, 2008.

${ }^{\dagger}$ The authors are with Institute of Computer \& Engineering, Beijing Jiaotong University, Beijing China.

a) E-mail: songhe_feng@163.com

b)E-mail: dxu@bjtu.edu.cn

c) E-mail: bjtulb@gmail.com

DOI: 10.1093/ietisy/e91-d.8.2203 manifold structure.

Not much attention has been paid to developing ranking measures that combine region-based image representation with manifold-ranking. Several efforts in this direction are proposed by [5] and [9]. Although these algorithms report satisfactory performances compared with [4], the most important problem with regard to region significance is omitted in these methods. This is because, in most cases, when a user submits a query image, he or she is only interested in some parts, instead of every part of the image. So how to measure the region's significance is of great importance in region-based image retrieval.

This observation prompts us to incorporate region saliency into the process of region-based image retrieval under a manifold-ranking framework. We argue that, in cases where region significance is considered in the manifoldranking based retrieval algorithm, both human perception and internal image manifold structure can be well encoded.

Specifically, we first propose an efficient and effective selective visual attention model with an image segmentation algorithm to measure the region significance. Then, a hierarchical graph model is built for the manifold-ranking algorithm which can well incorporate the factor of region significance. Concretely, the graph model consists of two levels. First, an abstract region-level weighted graph is defined. Note that "abstract" means it need not be explicitly built. Both region significance and visual feature vectors are considered to determine the edge weight between each region pair. Then, in the image-level graph, each image is considered a vertex, and the edge weights are determined based on the regions belonging to the image. The manifoldranking algorithm is adopted for the final label propagation. The benefits of the proposed hierarchical graph model lie in that not only the local semantic similarity is investigated but also regional saliency is considered.

The remainder of this paper is organized as follows. A visual attention model based region saliency computation method is proposed in Sect. 2. Then, Sect. 3 describes the proposed hierarchical graph model for the manifold-ranking algorithm where region saliency factor is encoded. Section 4 presents the experimental results and the performance evaluations. Finally, conclusions are given in Sect. 5 . 


\section{Visual Attention Based Image Analysis}

\subsection{Pixel-Level Saliency Map Computing}

The saliency value of each pixel is computed on three resolution scales of the image $(1,1 / 2$ and $1 / 4)$ separately. For a given color image $I$ with the size of $I_{M} \times I_{N}$ pixels, suppose $x$ is a given pixel in $I, \Phi_{x}$ is a size of $c \times c$ window centered at $x$ for the computation of contrast. Here we have $c=3$. Then we compute the feature contrast between $x$ and other pixels in $\Phi_{x}$. Many features can attract human's attention. Here we follow [1]'s method which considering color, intensity, and orientation features. So the saliency value for the pixel $x$ can be calculated as:

$$
S P(x)=\sum_{z \in \Phi_{x}}\left(\gamma_{C I} S_{C I}(x, z)+\gamma_{O} S_{O}(x, z)\right)
$$

where $z$ is the pixel belong to $\Phi_{x}, S_{C I}(x, z)$ and $S_{O}(x, z)$ denote the color-intensity contrast and orientation contrast between $x$ and $z$. Here, $\gamma_{C I}$ and $\gamma_{O}$ are the weighting coefficients and be set to 1 for simplicity. After that, we use a Gaussian filter to remove the noise points: $\hat{S P}(x)=$ $S P(x) \otimes G(x)$, where $G(x)$ is a Gaussian filter with the standard deviation $\sigma_{G}=1$. The same work can be done on other two resolution scales. After all the three-level saliency values are computed, we incorporate the results to form the final saliency value on the original resolution scale.

\subsection{Region-Level Saliency Definition}

Unlike the block-based uniform representation in [5], region-based representation is more effective for image retrieval due to the fact that human beings pay more attention to objects instead of blocks in an image. So in this section, the Normalized-Cut (NC) image segmentation algorithm [7] considering both color and texture information is utilized here. Note that using NC segmentation algorithm, the region number of images can be varied between each other.

Suppose the image $I$ is segmented into homogenous regions using the NC algorithm, i.e., $I=\left\{r_{i}\right\}, i=1, \cdots,|I|$, where $r_{i}$ is the $i$-th region and $|I|$ denotes the region number of $I$. For each segmented region $r_{i}$, the region saliency value can be calculated as follows:

$$
S R\left(r_{i}\right)=\sum_{x \in r_{i}} S P(x) / \operatorname{Area}\left(r_{i}\right)
$$

where $S P(x)$ denotes the saliency value of pixel $x$ which can be computed according to Eq. (1). Area $\left(r_{i}\right)$ is the area of $r_{i}$. Note that all the saliency energy values should be normalized from 0 to 1 .

According to the above definition, each region in the image will be given a saliency value which measures the importance factor during the similarity propagation. The examples of two images with extracted most salient regions are shown in Fig. 1. From left to right, the Figure illustrates the
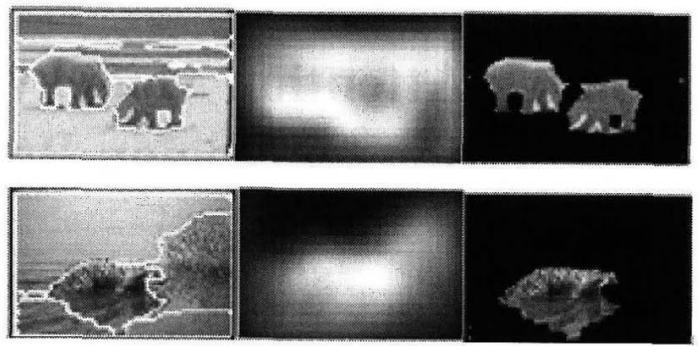

Fig. 1 Some results of extracted most salient regions.

segmented images, the saliency maps and the selected most salient regions. From the example, one can clearly see that the region salient value can well reflect the region's importance information and well fit human's perception.

\section{A Hierarchical Graph Representation for Manifold- Ranking Algorithm}

The manifold-ranking algorithm [6] is initially proposed as a semi-supervised learning framework, which aims to rank the data points or to predict the labels of unlabeled data points along their underlying manifold by analyzing their relationship in Euclidean space. Due to its good properties of feature representation, the manifold-ranking algorithm has been applied in image retrieval [4]. Here we shortly discuss the manifold-ranking algorithm at first and introduce the proposed hierarchical graph representation later.

\subsection{The Manifold-Ranking Algorithm}

In the scenario of image retrieval, assume a set of images $\chi=\left\{I_{1}, \cdots I_{q}, I_{q+1}, \cdots, I_{n}\right\} \subset \mathfrak{R}^{m}$ (here $m$ denotes the dimension for data features), the first $q$ images are the queries which form the query set, and the rest are to be ranked according to their relevance to the queries. Let $d: \chi \times \chi \rightarrow \mathfrak{R}$ denote a metric on $\chi$ which assigns to each pair of images $I_{i}$ and $I_{j}$ a distance $d\left(I_{i}, I_{j}\right)$, and $f: \chi \rightarrow \Re$ denote a ranking function which assigns to each image $I_{i}$ a ranking score $f_{i}$. Finally, a $n \times 1$ vector is defined to correspond to the ranking order for every label on the dataset and the initial vector is set as $Y=\left[y_{1}, y_{2}, \cdots, y_{n}\right]^{T}$, with $y_{i}=1$ if $I_{i}$ is a query image, and $y_{i}=0$ otherwise. The procedure of the manifold-ranking algorithm can be described below.

1. Sort the pair-wise distances among images in ascending order. Repeat connecting the two images with an edge according to the order until a connected graph is obtained.

2. Form the affinity matrix $W$ defined by $W_{i j}=$ $\exp \left[-d^{2}\left(I_{i}, I_{j}\right) / 2 \sigma^{2}\right]$ if there is an edge linking $I_{i}$ and $I_{j}$. Let $W_{i i}=0$.

3. Symmetrically normalize $W$ by $S=D^{-1 / 2} W D^{-1 / 2}$ in which $D$ is the diagonal matrix with $(i, i)$-element equal to the sum of the $i$-th row of $W$.

4. Iterate $f(t+1)=\alpha S f(t)+(1-\alpha) Y$ until convergence, where $\alpha$ is a parameter in $[0,1)$ and $f(0)=Y$. 
5. Let $f^{*}$ denote the limit of the sequence $\{f(t)\}$. Rank each image $I_{i}$ according to its ranking scores $f_{i}^{*}$.

\subsection{The Proposed Hierarchical Graph Representation}

By analyzing the above description of the classical manifold-ranking algorithm, one can find that the construction of affinity matrix $W$ (i.e., the graph) is at the heart of the algorithm. Existing block-based manifold-ranking algorithm took every part of the images to measure the similarity between images, and further formed the affinity matrix. Such manner is inconsistent with human perception since the user generally does not consider each region as equal interest (global-based method will only worsen the situation). In order to reflect such user's retrieval intention more accurately, inspired by [2], we propose the following rules in the manifold-ranking algorithm which can guide the new hierarchical graph construction.

Rule1. Salient regions should propagate more positive energy to nearby regions belonging to unlabeled or positively labeled images.

Rule2. An unlabeled region that is near salient regions should propagate positive energy to other nearby unlabeled regions, thereby strengthening the label of both their images.

The above criteria do guarantee that the label can be propagated according to human semantic understanding. Based on the existing two rules, we propose a hierarchical graph model for the manifold-ranking algorithm. Two levels of weighted graph are achieved: region-level and imagelevel, respectively.

\subsubsection{Region-Level Graph Construction}

We first define an abstract region-level weighted graph which need not be explicitly built. A vertex is placed in the region-level graph for each region. Each node is linked to its low-level visual features (e.g., the average HSV color features) and saliency energy which is computed in the Sect. 2. Now we define how the edge weights are computed for the region-level graph. We propose to create edges between regions based on their nearest neighbors. We compute the top $k$ nearest neighbors by calculating the similarity score between each region pair. We encode both the saliency energy and the visual features' similarity between regions into the edge weight. For vertices $r_{i}$ and $r_{j}$, we let

$$
w_{R}\left(r_{i}, r_{j}\right)=\frac{1}{2}\left(S R\left(r_{i}\right)+S R\left(r_{j}\right)\right) \exp \left(-d i s t^{2}\left(r_{i}, r_{j}\right)\right)
$$

where $S R\left(r_{i}\right)$ and $S R\left(r_{j}\right)$ are $r_{i}$ 's and $r_{i}$ 's region saliency values, respectively. $\operatorname{dist}\left(r_{i}, r_{j}\right)$ denotes the Euclidean distance between $r_{i}$ and $r_{j}$. Such edge weight definition encodes both visual similarity and region saliency into consideration. We have to point out that although such definition is apparently alike to [2], the intrinsical meanings are totally different. In [2] the value of $S R\left(r_{i}\right)$ denotes the likelihood that $r_{i}$ belongs to the user's retrieval intension, which has to be learned using both positive and negative images under the multi-instance learning algorithm. So such method needs a relevance feedback mechanism for the user to provide the training samples. Instead, in our method, the value of $S R\left(r_{i}\right)$ can be obtained directly based on the image's lowlevel features using the visual attention model and it reflects the saliency degree from the perspective of visual perception.

\subsubsection{Image-Level Graph Construction}

Now we use the region-level graph to define the truly built image-level graph that will be used as the affinity matrix $W$ by the manifold-ranking algorithm. A vertex is placed in the image-level graph for the query image and unlabeled images in the dataset. The edge weight between images $I_{u}$ and $I_{v}$ is the sum of all edge weights in the region-level graph between regions in $I_{u}$ and regions in $I_{v}$.

$$
w_{I}\left(I_{u}, I_{v}\right)=\sum_{r_{i} \in I_{u}, r_{j} \in I_{v}} w_{R}\left(r_{i}, r_{j}\right)
$$

Note that, here we do not take advantage of the well studied region matching strategy such as IRM [8]. Since this kind of approach would increase the computational cost, and our simple full-matching strategy has already taken the region saliency factor into account, we believe that the simple full-matching method is appropriate here. Besides, this strategy would not result in the imbalance problem which would not be allowed in manifold-ranking algorithm. After that, this graph definition can be further used in the classical manifold-ranking algorithm for the final image retrieval algorithm.

\section{Experimental Results}

We implement the proposed algorithm using MATLAB 6.5 on a PC with $3.0 \mathrm{G}$ Pentium IV CPU and $1 \mathrm{G}$ memory. To evaluate the performance of the proposed algorithm, we choose about 5,000 images of 50 categories from the COREL collection as our test image database. Each semantic category consists of 100 images. Alike [4], we use each image in the dataset as a query. The remaining 4999 images are considered as unlabeled images. The results are averaged over the 5,000 queries. The precision versus scope curve is used to evaluate the performance of various methods. We compared the performances of the following methods: (1) The global feature based manifoldranking algorithm [4], noted as "Global-MR"; (2) Retrieval using block based manifold-ranking algorithm [5], noted as "Block-MR"; (3) The well known SIMPLIcity system using Integrated region matching [8], noted as "IRM"; (4) The proposed hierarchical manifold-ranking algorithm, noted as "Hierarchical-MR".

Concretely, for the "Global-MR" method, we extract 64-d HSV color histogram and 6-d texture feature using wavelet transform to form the low-level features of each 


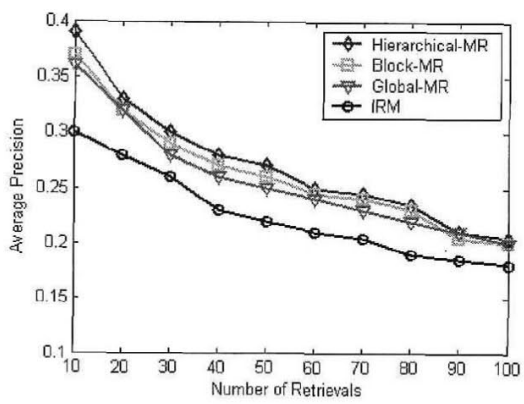

Fig. 2 The comparison of average precision rates respectively.

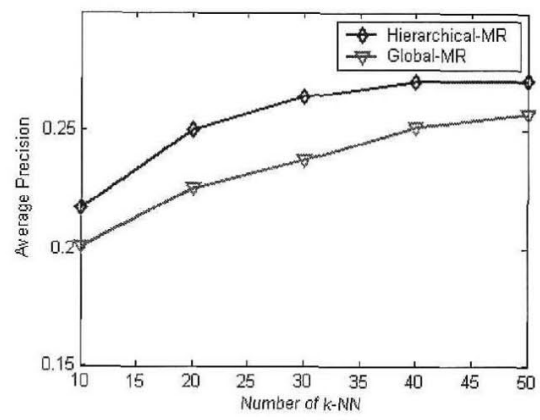

Fig. 3 The average precisions for different $k$ values in $k-N N$ based method.

image, and L1 distance is adopted to measure the similarity. For the "IRM" method, since the region matching strategy is the essential of the SIMPLIcity system, here we only adopt its region matching algorithm and still use the Normalized-Cut based image segmentation algorithm instead of the adoptive k-means algorithm used in SIMPLIcity system. According to the "Block-MR", it is the same with the algorithm proposed in [5].

In our experiment, we set $k=50$ and $\alpha=0.99$, where $k$ was used in the region-level graph construction. For the stage of region saliency computing, it can be finished offline in order not to affect the retrieval performance. The computing time of the region saliency calculation for each image in the dataset is $1.25 \mathrm{~s}$ (including $0.45 \mathrm{~s}$ for NC image segmentation method). The construction of affinity matrix can also be calculated offline which takes about 1.5 mins. Note that such construction need only be executed for just one time. As to the on-line cost, the retrieval stage takes about $0.65 \mathrm{~s}$ for each retrieval stage. The average semantic similarities of retrieval results are calculated by using the above methods, and the average precision rates are seen in Fig. 2. From the experiment, we can see that, since the impact of region saliency is considered under the hierarchical manifold-ranking algorithm, the average retrieval precision is better than the global feature based and block based methods, as well as the IRM method. Besides, Fig. 3 indicates the influence of the number of during the graph construction. Here only global based method is compared with the proposed algorithm. This shows that when $k$ is larger than 40 , the performance changes slightly.

\section{Conclusion and Future Work}

This paper presents a novel region-based image retrieval method which combines the attention model with the hierarchical graph representation. In contrast with existing algorithms, the proposed method takes region significance into consideration and uses a visual attention model for saliency computing. Then, a hierarchical graph representation which combines region-level with image-level similarity propagation steps is proposed and further used in the manifoldranking algorithm. The experimental results have demonstrated the satisfactory results of the proposed method. In future work, we are aiming at incorporating both multipleinstance learning and semi-supervised learning for the CBIR issue.

\section{Acknowledgement}

This work is supported by Chinese National Programs for High Technology Research and Development (Project No.2007AA01Z168).

\section{References}

[1] L. Itti, C. Koch, and E. Niebur, "A model of saliency-based visual attention for rapid scene analysis," IEEE Trans. Pattern Anal. Mach. Intell., vol.20, no.11, pp.1254-1259, 1998.

[2] R. Rahmani and S.A. Goldman, "MISSL: Multiple-instance semisupervised learning," Proc. ICML pp.705-712, Pittsburgh, PA, 2006.

[3] R. Datta, J. Li, and J.Z. Wang, "Content-based image retrieval - Approaches and trends of the new age," ACM MIR, 2005.

[4] J. He, M. Li, H.-J. Zhang, H. Tong, and C. Zhang, "Manifold-ranking based image retrieval," Proc. 12th ACM Conference on Multimedia, pp.9-16, 2004.

[5] X. Wan, "Content based image retrieval using manifold-ranking of blocks," Proc. IEEE Conference on Multimedia \& Expo, Beijing, China, 2007.

[6] D. Zhou, O. Bousquet, T.N. Lal, J. Weston, and B. Scholkopf, "Learning with local and global consistency," Proc. NIPS'03, 2003.

[7] J. Shi and J. Malik, "Normalized cuts and image segmentation," IEEE Trans. Pattern Anal. Mach. Intell., vol.22, no.8, pp.888-905, 2000.

[8] J.Z. Wang, J. Li, and G. Wiederhold, "SIMPLIcity: Semanticssensitive integrated matching for picture libraries," IEEE Trans. Pattern Anal. Mach. Intell., vol.23, no.9, pp.947-963, 2001.

[9] F. Li, Q. Dai, W. Xu, and G. Er, "Correlated probabilistic label propagation for region-based image retrieval," Proc. IEEE Conference on Acoustics, Speech, and Signal Processing, 2007. 